\title{
Ong eye speculum for glaucoma surgery
}

\author{
Keith Ong \\ University of Sydney, Australia; Royal North Shore Hospital, Australia; Dalcross Adventist \\ Hospital, Australia; and Ryde Hospital, Australia
}

In glaucoma surgery such as trabeculectomy, access to the superior sclera, conjunctiva and limbus is required. This can be a challenge in patients with small palpebral fissures. A superior rectus bridle suture or more commonly a traction suture through the superior peripheral cornea is used to rotate the eye to look down, thus exposing the superior sclera. Although the superior corneal traction suture causes minimal trauma, it may contribute to mild inflammation and can leave a small scar in the superior cornea.

The Ong eye speculum has a larger inferior inner blade of 12-15 $\mathrm{mm}$ compared to the standard speculum inner blades of about $5 \mathrm{~mm}$ (Fig. 1). The larger inferior blade pushes on the inferior conjunctival fornix and hence rotates the eyeball down (Fig. 2).

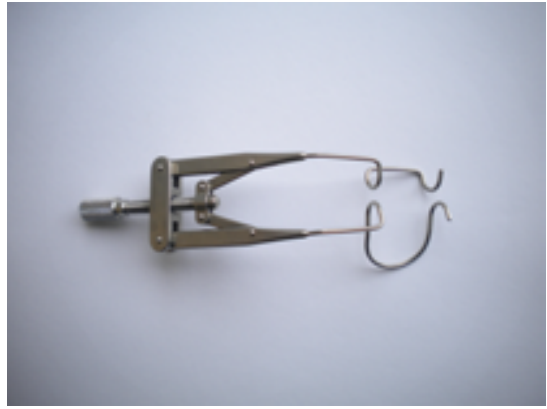

Fig. 1. Ong Speculum for the right eye.

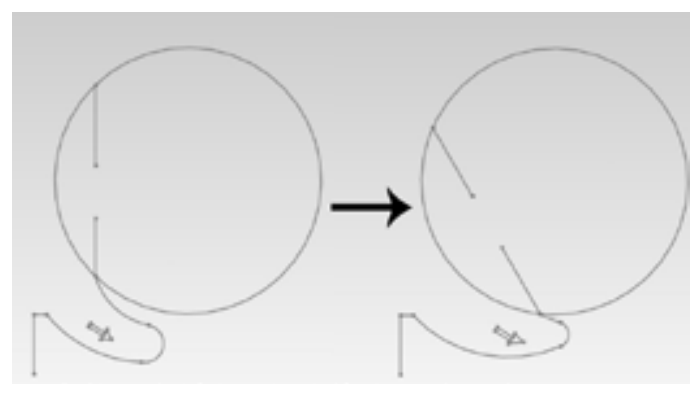

Fig. 2. Pushing on the inferior conjunctival fornix turns the eye down.

The inferior conjunctival fornix is $8-10 \mathrm{~mm}$ from the limbus ${ }^{1}$, and with the inferior blade being 12-15 $\mathrm{mm}$, it would rotate the eye down five to seven $\mathrm{mm}$, which in most cases would provide adequate exposure of the superior conjunctiva/sclera.

The superior arm has the standard five-mm size anterior and posterior blades to cradle the eyelid margin. The inferior arm has a five-mm anterior blade; and the posterior blade has a five-mm section to cradle the lid margin, and an inferior section of seven to ten $10 \mathrm{~mm}$ which is angled and curved posteriorly to push on the inferior fornix. This bend and curve prevents the tip of inferior blade of the speculum from impinging on the orbital floor and indenting the cornea-sclera; and also helps rotate the globe in the desired direction by pushing on the inferior conjunctival fornix. These two sections make up the 12-15 mm inferior posterior blade.

This new eye speculum design facilitates exposure of the superior sclera and limbus

Correspondence: Keith Ong, 2 Railway Avenue, Eastwood NSW 2122, Australia

E-mail: keith.ong@sydney.edu.au 
by rotating the eyeball to look down, without the need for a traction suture through the superior peripheral cornea, when doing trabeculectomy. It is also useful when doing procedures such as needling of filtration bleb and subconjunctival 5-Fluorouracil injections in which exposure of the superior conjunctiva is required. When there is akinesia of the globe after peribulbar, retrobulbar or subtenon's local anaesthetic, this speculum is useful as the patient is not able to voluntarily infraduct the eye.

The initial design was an open-wire eye speculum based on the Kratz-Barraquer design. The Ong speculum is now manufactured with the Lieberman speculum adjustable mechanism. This improves the functionality of the Ong speculum as it enables the amount of infraduction to be varied. The eye can be in primary position when the speculum is semi-open (Fig. 3). When the speculum is in open position, the inferior blade pushes on the inferior fornix and infraducts the eye (Fig. 4).

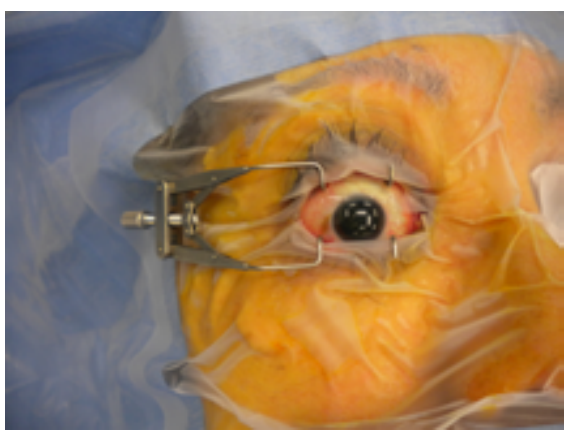

Fig. 3. Ong Speculum semi-open - eye in primary position.

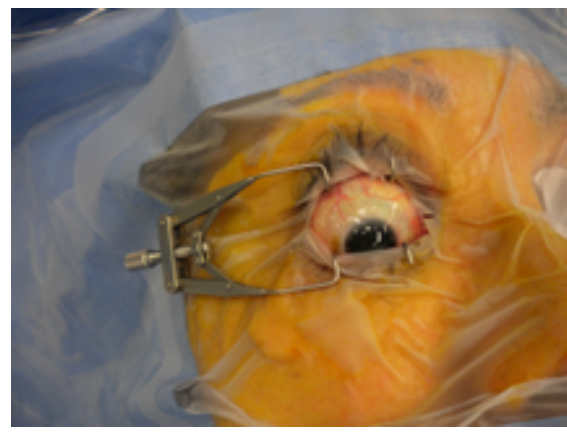

Fig. 4. Ong Speculum in open position - eye rotates down.

A range of sizes would enable the desired amount of infraduction to be achieved. The small Ong speculum is suitable for most trabeculectomy cases, and the large Ong speculum is useful when more infraduction is required, such as in glaucoma seton surgery. The small speculum may also be more appropriate for the smaller fornix of the oriental eye, while the larger speculum may suit the more capacious fornix of the Caucasian eye.

A full descriptive name of this eye speculum design would be Ong-Lieberman eye speculum. The Ong eye speculum is a descriptive nomenclature for an eye speculum with a larger inferior blade which pushes on the inferior conjunctival fornix to rotate the eyeball down.

The Ong Eye Speculum is manufactured by Amann Ophthalmic Instruments (Germany), and available through Designs for Vision (Australia). The catalogue numbers are E10460-50 (large left) and E10461-50 (large right), and E10460-40 (small left) and E10461-40 (small right). The author-designer has no financial interest in the product.

\section{References}

1. Wolff's Anatomy of the Eye and Orbit. $8^{\text {th }}$ Ed. London: Chapman and Hall, London 1997; p. 52. 